\title{
Equações de predição de valores energéticos de alimentos obtidas utilizando meta-análise e componentes principais
}

\author{
Prediction equations of energetic values of feedstuffs obtained using \\ meta-analysis and principal components
}

\begin{abstract}
Flávia Cristina Martins Queiroz Mariano ${ }^{\mathrm{I}}$ Renato Ribeiro de Lima $^{\mathrm{I}}$ Paulo Borges Rodrigues ${ }^{\mathrm{II}}$ Renata Ribeiro AlvarengaII Germano Augusto Jerônimo do Nascimento ${ }^{\text {III }}$
\end{abstract}

\section{RESUMO}

\begin{abstract}
Neste estudo, foi proposta a utilização da análise de componentes principais, na formação de grupos homogêneos de artigos científicos, a serem considerados em uma meta-análise. Nessa meta-análise, foram utilizados resultados referentes à composição química e energia metabolizável aparente corrigida pelo balanço de nitrogênio (EMAn) de alimentos para aves, na obtenção de equações de predição da EMAn. Foram considerados 293 experimentos com resultados já publicados. Dados provenientes de ensaios metabólicos foram utilizados na validação das equações de predição obtidas, sendo que estas apresentaram resultados semelhantes às disponiveis na literatura. No procedimento de meta-análise, a formação de grupos homogêneos de resultados experimentais, que é uma das maiores dificuldades, foi facilitada com a utilização de componentes principais, uma vez que não houve a necessidade de determinar variáveis ou fatores a serem considerados nessa classificação. Assim, tem-se uma forma rápida e eficiente de definir tais grupos.
\end{abstract}

Palavras-chave: composição química, energia metabolizável, validação de equações.

\section{ABSTRACT}

The purpose of this study was to develop a metaanalysis study by using the principal components analysis to obtain homogeneous groups of experimental results. In the process of the meta-analysis, it was considered data from 293 experiments carried out in Brazil. Prediction equations were obtained to estimate the nitrogen-corrected apparent metabolizable energy (AMEn) of poultry feedstuffs. Data from metabolic trials were used to validate the prediction equations obtained, which were similar than other available equations in the literature. One of the problems in meta-analysis is the determination of the homogeneous groups of experiments and, this problem was eliminated by using principal components, since there was no need to establish variables or factors to be considered in this classification.

Key words: chemical composition, metabolizable energy, validation of equations.

\section{INTRODUÇÃO}

A diversidade de alimentos e subprodutos utilizados na alimentação de frangos de corte exige o conhecimento preciso da composição química e valores de energia metabolizável (EM) desses alimentos, possibilitando a formulação de dietas equilibradas nutricional e economicamente. Uma forma rápida de determinar os valores de EM consiste na utilização de equações de predição, estabelecidas em função da composição química deles, normalmente de fácil e rápida obtenção.

Existem, à disposição na literatura, vários trabalhos de pesquisa, em que se estabeleceram equações de predição para os valores energéticos de uma série de alimentos (ZHAO et al., 2008; WAN et al., 2009). Porém, os resultados obtidos utilizando-se essas equações não têm sido satisfatórios. Com o intuito de melhorá-los, pode-se utilizar o princípio da meta-análise. Um dos objetivos da meta-análise é a obtenção de novos resultados, a partir da síntese de n observações distintas, porém relacionadas entre si (LOVATTO et

\footnotetext{
'Departamento de Ciências Exatas, Universidade Federal de Lavras (UFLA), CP 3037, 37200-000, Lavras, MG, Brasil. E-mail: flaviaqz@gmail.com. *Autor para correspondência.

"Departamento de Zootecnia, UFLA, Lavras, MG, Brasil.

II'Departamento de Zootecnia, Universidade Federal do Ceará (UFC), Fortaleza, CE, Brasil.
} 
al., 2007). Assim, avalia-se um número maior de trabalhos, o que garante a obtenção de resultados mais consistentes.

Segundo LOVATTO et al. (2007), algumas etapas necessárias no desenvolvimento de uma metaanálise são: a) definição do objetivo do trabalho, do tema da pesquisa; b) sistematização das informações; c) codificação dos dados; d) filtragem dos dados; e) análise dos dados incluídos na base; f) formação de grupos; g) ponderações; e h) escolha de um modelo estatístico. Embora os estudos considerados envolvam um mesmo assunto, na meta-análise, é importante que grupos homogêneos destes sejam formados e a variabilidade existente entre eles seja modelada e incorporada na análise. No entanto, a formação de grupos homogêneos é uma das maiores dificuldades na meta-análise, podendo ser feita por combinação de níveis de fatores, análises de agrupamento, etc.

NASCIMENTO et al. (2009, 2011a, 2011b) estabeleceram equações de predição de EMAn utilizando o princípio da meta-análise, definindo 24 grupos homogêneos pela combinação de três fatores, que influenciavam na variabilidade da EMAn: sexo (macho, fêmea e mistos); idade (1를 e $2^{\underline{a}}$ semanas de vida; 3 e 4a semanas; 5a e $6^{\underline{a}}$ semanas; acima ou indefinido); e metodologia empregada (coleta total CT; alimentação forçada + CT).

Neste trabalho, foi proposta a utilização da análise de componentes principais como uma forma mais simples e direta de agrupamento dos resultados experimentais mais homogêneos, a serem considerados na meta-análise. Essa meta-análise foi desenvolvida para a obtenção de equações de predição para energia metabolizável aparente corrigida pelo balanço de nitrogênio (EMAn) em função da composição química de alimentos para aves. Tais equações obtidas foram validadas, utilizando-se dados provenientes de ensaios metabólicos, e, posteriormente, foram comparadas com outras já publicadas na literatura.

\section{MATERIAL E MÉTODOS}

Os dados utilizados na obtenção das equações de predição, via meta-análise, foram catalogados por NASCIMENTO et al. (2009, 2011a, 2011 b). Esses dados referem-se a valores de energia metabolizável aparente corrigida pelo balanço de nitrogênio (EMAn) e composição química (proteína bruta-PB, extrato etéreo-EE, matéria mineral-MM, fibra bruta-FB, fibra em detergente neutro-FDN e fibra em detergente ácido-FDA) de alimentos concentrados energéticos e proteicos de origem vegetal, usualmente utilizados na formulação das rações avícolas. Neste estudo, foram utilizados diferentes resultados experimentais, realizados no Brasil, classificados em três categorias de alimentos: 197 trabalhos envolvendo equações de predição da EMAn de alimentos energéticos, 67 de soja + subprodutos (proteicos) e 293 de concentrados em geral (energéticos + proteicos).

$\mathrm{Na}$ formação de grupos homogêneos de resultados experimentais, foi proposta a utilização da técnica multivariada de componentes principais como método de agrupamento. Com o uso dessa técnica, o número de grupos a serem formados não é explicitamente definido como no caso em que os grupos são formados por combinação de níveis de fatores. Assim, para identificar qual é o número mais adequado de grupos homogêneos foram considerados $2,3,4,5,6,7,8,9,10,20,25,30,40$ e 50 grupos. Além disso, foi considerada a situação na qual não se utiliza a meta-análise, ou seja, sem a formação de grupos. Portanto, foram realizadas 45 meta-análises, isto é, 15 critérios para a formação de grupos para cada uma das três categorias de alimentos.

Os passos desde a formação de grupos até a obtenção das equações de predição, em cada metaanálise, foram: (i) efetuar a análise de componentes principais, considerando as variáveis EMAn, PB, EE, MM, FB, FDN e FDA, obtendo-se o valor do primeiro componente principal (PC1) para cada um dos resultados experimentais; (ii) ordenar os valores referentes aos $\mathrm{PC}$; (iii) definir o número de grupos; (iv) estabelecer os quantis que delimitam os grupos homogêneos. Por exemplo: definindo-se 5 grupos, temse os quantis 0,$20 ; 0,40 ; 0,60$ e 0,80 . Abaixo do quantil 0,20 , estão os $20 \%$ dos resultados experimentais com os menores escores de $\mathrm{PC} 1$, que compõem o primeiro grupo. Entre o quantil 0,20 e 0,40, estão os próximos $20 \%$ dos resultados experimentais com menores valores de PC1, compondo o segundo grupo e assim por diante; (v) identificar os resultados experimentais dentro de cada grupo estabelecido; (vi) calcular a variância da EMAn observada nos resultados experimentais de cada grupo; (vii) obter o fator de ponderação que, neste trabalho, foi definido como o inverso da variância dentro de cada grupo; (viii) obter a equação de regressão estimada, utilizando o método dos mínimos quadrados ponderados, sendo os fatores de ponderação definidos em (vii).

Para o ajuste das equações, foi proposto o modelo de regressão linear múltipla $Y_{i}=\beta_{0}+\beta_{1} X_{i 1}+\beta_{2} X_{i 2}+\ldots+\beta_{6} X_{i 6}+\varepsilon_{i}$, em que: $\mathrm{Y}_{\mathrm{i}}$ refere-se ao valor da EMAn do alimento, no i-ésimo estudo; $\mathrm{X}_{\mathrm{i} 1}, \ldots, \mathrm{X}_{\mathrm{i} 6}$ representam as variáveis de composição química do alimento, no i-ésimo estudo, sendo respectivamente $\mathrm{PB}, \mathrm{MM}$, EE, FB, FDN e FDA 
e $\varepsilon_{\mathrm{i}}$ é o erro associado na i-ésima observação, assumido normal e independentemente distribuído, com média $0 \mathrm{e}$ variância $\sigma_{i}^{2}$. O método stepwise foi adotado, sendo o critério de Akaike (AIC) utilizado na inclusão e exclusão de variáveis no modelo. Após a aplicação do stepwise, se alguma variável foi não significativa a $10 \%$, pelo teste $\mathrm{t}$, esta foi retirada do modelo.

Para efeito de seleção das equações obtidas neste trabalho que apresentaram melhores ajustes, utilizou-se, simultaneamente, o valor de AIC com os valores dos critérios de validação de cada meta-análise efetuada.

As equações que apresentaram melhores ajustes neste estudo, bem como equações de predição da EMAn de alimentos para aves recomendadas na literatura (RODRIGUES et al., 2001; 2002, NAGATA et al., 2004; ZONTA et al., 2004; NASCIMENTO et al., 2009, 2011a, 2011b) foram submetidas à validação. Nesta validação, foram utilizados dados provenientes de dois ensaios metabólicos, conduzidos por ALVARENGA et al. (2011). Nesses ensaios, foram utilizados nove ingredientes proteicos e nove energéticos, fornecidos a pintos machos da linhagem Cobb $500^{\circledR}$, utilizando o método tradicional de coleta total de excretas.

Para efeito de comparação, foram confrontados os resultados das equações selecionadas neste estudo e na literatura (Tabelas 1 e 2), considerando diferentes critérios de validação, descritos por TEDESCHI (2006). Um critério consistiu no ajuste do modelo de regressão linear simples $Y_{i}=\beta_{0}+\beta_{1} X_{i}+\varepsilon_{i}$, em que: $\mathrm{Y}_{\mathrm{i}}$ é a EMAn do alimento $\mathrm{i}$ obtida no ensaio metabólico; $\mathrm{X}_{\mathrm{i}}$ é o valor predito da EMAn, utilizando-se uma equação selecionada; e $\varepsilon_{i}$ é o erro tal que $\varepsilon_{i} \sim \mathrm{N}\left(0, \sigma^{2}\right)$. As hipóteses $\mathrm{H}_{0}: \beta_{0}=0$ e $\mathrm{H}_{0}$ : $\beta_{1}=1$ foram verificadas utilizando-se o teste $\mathrm{t}$ de Student, o que corresponde à hipótese de igualdade entre valores preditos e observados. Outro critério foi o coeficiente de correlação de concordância (CCC) entre os valores observados $\left(\mathrm{Y}_{\mathrm{i}}\right)$ e os valores preditos de EMAn $\left(\mathrm{X}_{\mathrm{i}}\right)$, proposto por LIN (1989). O CCC verifica, simultaneamente, se o modelo apresenta resultado mais acurado e preciso.

Toda rotina computacional utilizada neste trabalho foi desenvolvida no software livre R ( R Development Core Team, 2011).

\section{RESULTADOS E DISCUSSÃO}

Equações de predição para EMAn selecionadas, obtidas neste estudo

Para a categoria dos alimentos energéticos, $\mathrm{o}$ primeiro componente principal (PC1) de cada trabalho explicou $69 \%$ da variação total. Em ambas as categorias dos alimentos proteicos (soja+subprodutos) e na dos concentrados em geral, $52 \%$ da variação total foi explicada pelo uso dos PC1. Quanto maior a

Tabela 1 - Valores dos critérios de seleção e validação das equações de predição para alimentos energéticos.

\begin{tabular}{|c|c|c|c|c|c|c|}
\hline \multirow{2}{*}{ Equação (Eq.) } & \multirow{2}{*}{ Fonte $(*)$} & \multirow[b]{2}{*}{$\mathrm{R}^{2}(\%)$} & \multirow[b]{2}{*}{ AIC } & \multirow[b]{2}{*}{$\mathrm{p}$-valor $\beta_{0}$} & \multirow[b]{2}{*}{$\mathrm{p}$-valor $\beta_{1}$} & \multirow[b]{2}{*}{$\mathrm{CCC}$} \\
\hline & & & & & & \\
\hline 1 & Energéticos (10 grupos) & 53,80 & 37,870 & 0,454 & 0,224 & 0,990 \\
\hline 2 & Energéticos (20 grupos) & 47,46 & 90,441 & 0,198 & 0,403 & 0,987 \\
\hline 3 & Concentrados (10 grupos) & 71,00 & $-42,219$ & 0,190 & 0,177 & 0,985 \\
\hline 4 & Concentrados (20 grupos) & 74,50 & $-3,054$ & 0,075 & 0,075 & 0,982 \\
\hline 5 & NASCIMENTO et al. (2011a) & 81,00 & $-291,527$ & 0,073 & 0,029 & 0,994 \\
\hline 6 & NASCIMENTO et al. (2011a) & 81,00 & $-285,706$ & 0,064 & 0,037 & 0,992 \\
\hline 7 & NASCIMENTO et al. (2009) & 84,00 & $-512,111$ & 0,169 & 0,180 & 0,988 \\
\hline 8 & NASCIMENTO et al. (2009) & 83,00 & $-508,325$ & 0,293 & 0,339 & 0,985 \\
\hline 9 & RODRIGUES et al. (2001) & 96,00 & - & 0,767 & 0,753 & 0,987 \\
\hline 10 & RODRIGUES et al. (2001) & 95,00 & - & 0,085 & 0,054 & 0,841 \\
\hline 11 & NAGATA et al. (2004) & 92,00 & - & 0,181 & 0,182 & 0,781 \\
\hline
\end{tabular}

(*) Eq. 1: 4215,773+51,211EE-193,436MM-28,034FDN. $\quad$ Eq. $\quad 2: \quad 4235,378-11,487 \mathrm{~PB}+40,232 \mathrm{EE}-138,642 \mathrm{MM}-42,872 \mathrm{FB}-$ 21,738FDN+11,743FDA. Eq. 3: 4144,914+53,137EE-204,644MM-26,214FB-20,260FDN. Eq. 4: 4164,187+51,006EE-197,663MM35,689FB-20,593FDN. Eq. 5: 4371,18-26,48PB+30,65EE-126,93MM-52,26FB-25,14FDN+24,40FDA. Eq. 6: 4205,23+30,58EE130,35MM-58,29FB-28,31FDN+16,71FDA. Eq. 7: 4101,33+56,28EE-232,97MM-24,86FDN+10,42FDA. Eq. 8: 4095,41+56,84EE225,26MM-22,24FDN. Eq. 9: 4281,60-39,97FDN-72,90MM. Eq. 10: 4354,80-112,05FB-151,74MM. Eq. 11: 4021,80-227,55MM. As equações 1 a 4 foram obtidas, neste trabalho, utilizando-se a aplicação de meta-análise e componentes principais. As equações 3 , 4 , 7 e 8 foram obtidas considerando os alimentos concentrados e as demais equações, levando em conta alimentos energéticos. $\left({ }^{1}\right) \mathrm{R}^{2}=\operatorname{coeficiente}$ de determinação; $\mathrm{AIC}=$ critério de Akaike; $\mathrm{CCC}=$ coeficiente de correlação de concordância.

Ciência Rural, v.42, n.9, set, 2012. 
Tabela 2 - Valores dos critérios de seleção e validação das equações de predição para soja + subprodutos.

\begin{tabular}{|c|c|c|c|c|c|c|}
\hline \multirow{2}{*}{ Eq. } & \multirow{2}{*}{ Fonte $(*)$} & \multicolumn{5}{|c|}{ Critérios estatísticos $\left({ }^{1}\right)$} \\
\hline & & $\mathrm{R}^{2}(\%)$ & AIC & p-valor $\beta_{0}$ & p-valor $\beta_{1}$ & $\mathrm{CCC}$ \\
\hline 1 & Soja+subprodutos(10grupos) & 86,90 & 39,685 & 0,700 & 0,533 & 0,880 \\
\hline 2 & Soja + subprodutos(20grupos) & 84,10 & 98,979 & 0,014 & 0,002 & 0,959 \\
\hline 3 & Concentrados (10 grupos) & 71,00 & $-42,219$ & 0,708 & 0,737 & 0,980 \\
\hline 4 & Concentrados (20 grupos) & 74,50 & $-3,054$ & 0,710 & 0,712 & 0,973 \\
\hline 5 & NASCIMENTO et al.(2011b) & 81,00 & $-94,118$ & 0,978 & 0,966 & 0,750 \\
\hline 6 & NASCIMENTO et al. (2009) & 84,00 & $-512,111$ & 0,983 & 0,888 & 0,995 \\
\hline 7 & NASCIMENTO et al. (2009) & 83,00 & $-508,325$ & 0,992 & 0,864 & 0,991 \\
\hline 8 & RODRIGUES et al. (2002) & 93,00 & - & 0,474 & 0,336 & 0,209 \\
\hline 9 & ZONTA et al. (2004) & 92,00 & - & 0,000 & 0,002 & $-0,030$ \\
\hline 10 & ZONTA et al. (2004) & 90,00 & - & 0,415 & 0,539 & 0,017 \\
\hline
\end{tabular}

(*) Eq. 1: 3383, 589+51, 412EE-139, 617MM-15, 474FDA. Eq. 2: 1322, 267+33, 894PB+73, 606EE-84, 217MM+47, 200FB-29, 936FDN. Eq. 3: 4144, 914+53, 137EE-204, 644MM-26, 214FB-20, 260FDN. Eq. 4: 4164, 187+51, 006EE-197, 663MM-35, 689FB-20, 593FDN. Eq 5: 2707, 71+58, 63EE-16, 06FDN. Eq. 6: 4101, 33+56, 28EE-232, 97MM-24, 86FDN+10, 42FDA. Eq. 7: 4095, 41+56, 84EE-225, 26MM22, 24FDN. Eq. 8: 2822, 2-90, 13FB+49, 96EE. Eq. 9: -822, 33+69, 54PB-45, 26FDA+90, 81EE. Eq. 10: 2723, 05-50, 52FDA+60, 40EE As equações 1 a 4 foram obtidas, neste trabalho, utilizando-se a aplicação de meta-análise e componentes principais. As equações $3,4,6$ e 7 foram obtidas considerando como base de dados os alimentos concentrados e as demais equações, levando em conta alimentos soja + subprodutos. $\left({ }^{1}\right) \mathrm{R}^{2}=$ coeficiente de determinação; AIC = critério de Akaike; $\mathrm{CCC}=$ coeficiente de correlação de concordância.

porcentagem de explicação da variabilidade pelo PC1, melhor será o processo de formação de grupos de resultados experimentais homogêneos.

Para os alimentos energéticos, os valores dos critérios utilizados na seleção e validação das equações de predição estão representados na figura 1 . À medida que foi utilizado um maior número de grupos, os valores de AIC aumentaram rapidamente, até próximo de cinco grupos (Figura 1a). A partir daí, observa-se um aumento menos expressivo com uma tendência de se estabilizar. Optou-se por utilizar o AIC, pois, apesar do critério do coeficiente de determinação $\left(\mathrm{R}^{2}\right)$ (Figura 1b) ser largamente utilizado para a escolha do melhor modelo, ele é muito influenciado pelo número de parâmetros do modelo estatístico considerado. Verificase que, na figura $1 \mathrm{c}$, com o aumento do número de grupos, principalmente a partir de 20 grupos, os valores de CCC diminuem. Isso não é desejado, pois, se a partir de uma equação obtêm-se valores preditos mais próximos dos valores observados de EMAn, o CCC tende a um. Na figura 1d, têm-se os p-valores do teste $\mathrm{t}$, correspondentes aos resultados da validação, utilizando o ajuste da equação linear simples $\left(\mathrm{H}_{0}\right.$ : $\beta_{0}=$ 0 e $\left.\mathrm{H}_{0}: \beta_{1}=1\right)$. Nota-se que, nas situações em que foram considerados 1, 3, 6, 8 e 9 grupos, essas hipóteses foram rejeitadas. Assim, analisando-se concomitantemente o AIC, o CCC e os p-valores, conclui-se que as equações de predição da EMAn que tiveram os melhores ajustes para os concentrados energéticos foram aquelas obtidas com a definição de 10 e 20 grupos, pois, com esses números de grupos, para o conjunto de dados utilizados, têm-se melhores resultados em relação aos critérios utilizados.

Nas outras duas situações, nas quais consideram os 293 resultados experimentais de alimentos concentrados e os 67 de soja+subprodutos, foram realizados procedimentos semelhantes aos dos alimentos energéticos. Coincidentemente, as equações de predição com 10 e 20 grupos no processo de estimação, para os dados utilizados, foram consideradas as melhores. Assim, as equações que apresentaram melhores ajustes, segundo o critério AIC e os resultados da validação, estão apresentadas nas quatro primeiras linhas da tabela 1, para alimentos energéticos, e, nas quatro primeiras linhas da tabela 2, para soja + subprodutos.

Comparação de equações de predição via validação com ensaio metabólico

Alimentos energéticos e concentrados

As equações de predição para EMAn de alimentos energéticos, utilizadas na comparação, foram aquelas descritas na tabela 1 . Observa-se que essas equações são obtidas considerando-se diferentes bases de dados (apenas alimentos energéticos ou 


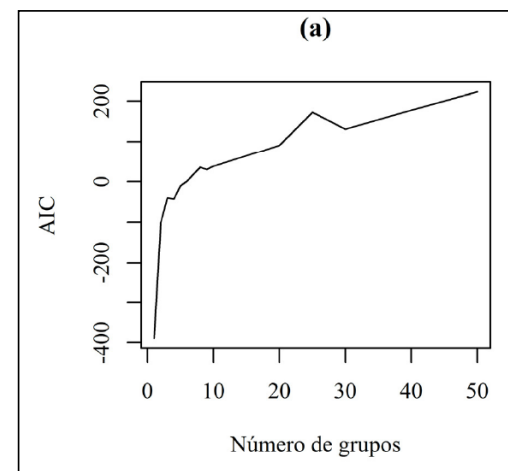

(c)

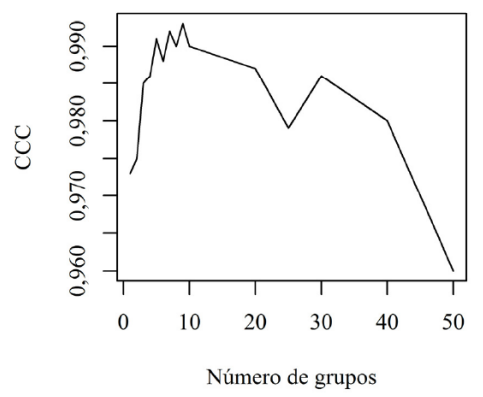

(b)

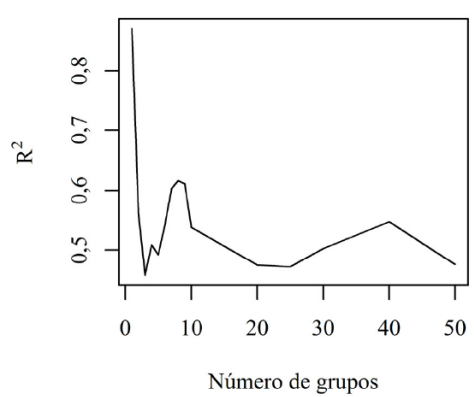

(d)

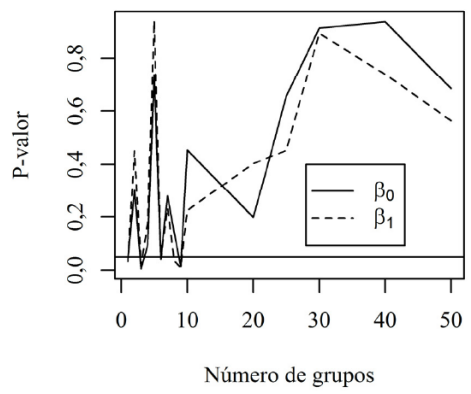

Figura 1 - Valores do (a) critério de Akaike, (b) coeficiente de determinação, (c) coeficiente de correlação de concordância e (d) p-valores de $\beta_{0}$ e $\beta_{1}$ determinados no ajuste das equações de predição para EMAn de alimentos energéticos, obtidos neste trabalho, em função do número de grupos formados.

concentrados em geral), mas que foram utilizadas na validação da EMAn de alimentos energéticos.

Na tabela 1, observa-se que, com exceção das equações 5 e 6 , as equações consideradas para alimentos energéticos não apresentam diferenças significativas entre os valores ajustados e os observados no ensaio metabólico. Ao analisar o coeficiente de correlação de concordância (CCC), notase que, até a equação 9, utilizada nessa comparação, todas possuem valores semelhantes de CCC, sendo todos próximos de um. Também, verifica-se que, apesar de as equações 1, 2, 3, 8 e 9 não apresentarem, em sua maioria, coeficientes de determinação $\left(\mathrm{R}^{2}\right)$ mais altos, estas possuem os maiores $p$-valores para os parâmetros da regressão linear simples. Então, com a análise simultânea desses critérios, conclui-se que as equações 1, 2, 3, 4, 7, 8 e 9 descreveram com acurácia e precisão os valores da EMAn obtidos no ensaio metabólico para alimentos energéticos.

Portanto, as equações obtidas neste trabalho apresentaram resultados semelhantes às disponíveis na literatura (RODRIGUES et al., 2001; NASCIMENTO et al., 2009, 2011a). Em NASCIMENTO et al. (2009, 2011a), assim como neste estudo, teve-se um baixo custo na obtenção de tais equações, pois foi utilizada meta-análise ao invés de executarem experimentos específicos como em RODRIGUES et al. (2001). Porém, o desenvolvimento da meta-análise foi facilitado neste estudo, uma vez que não houve a necessidade de definir fatores mais adequados para a formação de grupos homogêneos de resultados experimentais, como em NASCIMENTO et al. (2009, 2011a).

\section{Soja + subprodutos e concentrados}

As equações de predição para EMAn de soja+subprodutos, utilizadas na comparação, estão apresentadas na tabela 2. A comparação foi desenvolvida entre as diferentes equações de predição obtidas e aquelas adquiridas em trabalhos de outros autores, análogo ao feito para os alimentos energéticos e concentrados.

Pelos valores dos critérios apresentados na tabela 2, observa-se que as únicas equações de predição para EMAn para soja+subprodutos, em que as hipóteses estabelecidas foram rejeitadas durante a validação, foram as equações 2 e 9 . As demais não 
apresentaram diferenças significativas entre os valores ajustados e os observados no ensaio metabólico. Verifica-se, também, que, por possuírem valores próximos de CCC tendendo a um, as equações 2, 3, 4, 6 e 7 apresentam os melhores ajustes dentre as equações comparadas. No entanto, as equações 3, 4, 6 e 7 não apresentaram os maiores coeficientes de determinação $\left(\mathrm{R}^{2}\right)$, porém possuem maiores $p$-valores. Contudo, se fosse considerado só o coeficiente de determinação como critério único de seleção, o melhor ajuste considerado seria o da equação 8 , a qual possui valor de CCC igual a 0,21 . No decorrer de todo este trabalho, verifica-se que o $\mathrm{R}^{2}$ não é indicado a ser usado como critério único de seleção, pois o seu resultado não refletiria o que foi concluído na análise concomitante dos demais critérios. Portanto, diante desses resultados, recomenda-se, sempre que possível, a utilização conjunta dos critérios de seleção e validação de modelos. Assim, fazendo a análise simultânea desses critérios, conclui-se que as equações 3, 4, 6 e 7 descreveram com maior acurácia e precisão os valores da EMAn obtidos no ensaio metabólico para soja+subprodutos.

Portanto, as equações obtidas neste trabalho apresentaram resultados semelhantes às disponíveis em NASCIMENTO et al. (2009, 2011b). Análogo ao discutido na comparação para alimentos energéticos, a formação de grupos homogêneos foi facilitada através da técnica multivariada de componentes principais, uma vez que não é muito simples determinar quais os fatores que causam variabilidade entre os experimentos e que devam ser considerados na identificação de resultados experimentais homogêneos.

As equações ajustadas e selecionadas neste trabalho, cujos valores de $\mathrm{R}^{2}$ foram altos, estão sendo testadas por meio de ensaios metabólicos realizados com frangos de corte em fase de crescimento, conforme trabalho realizado por ALVARENGA et al. (2011).

\section{CONCLUSÃO}

As equações de predição obtidas neste trabalho apresentaram resultados semelhantes a outras propostas na literatura. Porém, a formação de grupos homogêneos de trabalhos, que é uma das maiores dificuldades na meta-análise, foi facilitada com a utilização da técnica de componentes principais. Assim, a utilização dessa técnica é viável por ser uma forma mais simples e direta de agrupamento dos trabalhos mais homogêneos, sem a necessidade da determinação de fatores coerentes para esse agrupamento como proposto em outros trabalhos.

\section{AGRADECIMENTO}

Os autores agradecem o apoio financeiro do Conselho Nacional de Desenvolvimento Científico e Tecnológico (CNPq) e da Coordenação de Aperfeiçoamento de Pessoal de Nível Superior (CAPES)

\section{REFERÊNCIAS}

ALVARENGA, R.R. et al. Energetic values of feedstuffs for broilers determined with in vivo assays and prediction equations. Animal Feed Science Technology, Amsterdam, v.168, Issues 3-4, p.257266, set. 2011. Disponível em: $<$ http://www.sciencedirect.com/ science/article/pii/S0377840111002276>. Acesso em: 22 set. 2011. doi:10.1016/j.anifeedsci.2011.04.092.

LIN, L. A concordance correlation coefficient to evaluate reprodutibility. Biometrics, Washington, v.45, n.1, p.255268, mar. 1989. Disponível em: <http://www.jstor.org/stable/ 2532051>. Acesso em: 13 set. 2009.

LOVATTO, P.A. et al. Meta-análise em pesquisas científicas: enfoque em metodologias. Revista Brasileira de Zootecnia, Viçosa, v.36, supl., p.285-294, jul. 2007. Disponível em: $<$ http://www.scielo.br/ scielo.php?pid $=\mathrm{S} 1516-35982007001000026 \&$ script $=$ sci_arttext $>$. Acesso em: 11 out. 2008. doi: 10.1590/S1516-35982007001000025.

NAGATA, A.K. et al. Energia metabolizável de alguns alimentos energéticos para frangos de corte, determinada por ensaios metabólicos e por equações de predição. Ciência e Agrotecnologia, Lavras, v.28, n.3, p.668-677, maio/jun. 2004 Disponível em: <http://www.scielo.br/scielo.php?pid=S1413$70542004000300025 \&$ script $=$ sci arttext $>$. Acesso em: 20 jun. 2009. doi: $10.1590 / \mathrm{S} 1413-70542004000300024$.

NASCIMENTO, G.A.J. et al. Equações de predição para estimar os valores energéticos de alimentos concentrados de origem vegetal para aves utilizando a metanálise. Revista Brasileira de Zootecnia, Viçosa, v.38, n.7, p.1265-1271, dez. 2009. Disponível em: <http:// www.scielo.br/scielo.php?script $=$ sci_arttext\&pid $=\mathrm{S} 1516$ 35982009000700015>. Acesso em: 10 jan. 2010. doi: 10.1590/ S1516-35982009000700014.

NASCIMENTO, G.A.J. et al. Equações de predição para estimar valores da energia metabolizável de alimentos concentrados energéticos para aves utilizando meta-análise. Arquivo Brasileiro de Medicina Veterinária e Zootecnia, Belo Horizonte, v.63, n.1, p.222-230, fev. 2011a. Disponível em: $<$ http://www.scielo.br/scielo.php?script=sci_arttext\&pid=S010209352011000100032>. Acesso em: 10 set. 2011. doi: 10.1590/ S0102-09352011000100032.

NASCIMENTO, G.A.J. et al. Equações de predição para estimar os valores da EMAn de alimentos protéicos para aves utilizando a meta-análise. Revista Brasileira de Zootecnia, Viçosa, v.40, n.10, p.2172-2177, out. 2011b. Disponível em: <http:/ /www.scielo.br/scielo.php?script $=$ sci_arttext\&pid $=\mathrm{S} 1516$ 35982011001000016>. Acesso em: 15 dez. 2011. doi: $10.1590 / \mathrm{S} 1516-35982011001000016$.

R DEVELOPMENT CORE TEAM. R: a language and enviroment for statistical computing. Viena: R Foundation for Statistical Computing, 2011. Software.

RODRIGUES, P.B. et al. Valores energéticos do milheto, do milho e subprodutos do milho, determinados com frangos de corte e galos 
adultos. Revista Brasileira de Zootecnia, Viçosa, v.30, n.6, p.1767-1778, nov./dez. 2001. Disponível em: <http:/www.scielo.br/ scielo.php?pid=S1516-35982001000700015\&script $=$ sci_arttext $>$. Acesso em: 23 jun. 2009. doi: 10.1590/S1516-35982001000700015.

RODRIGUES, P.B. et al. Valores energéticos da soja e subprodutos da soja, determinados com frangos de corte e galos adultos. Revista Brasileira de Zootecnia, Viçosa, v.31, n.4, p.1771-1782, jul./ ago. 2002. Disponível em: <http://www.scielo.br/ scielo.php?pid=S1516-35982002000700020\&script $=$ sci_arttext $>$. Acesso em: 23 jun. 2009. doi: 10.1590/S1516-35982002000700020.

TEDESCHI. L.O. Assessment of the adequacy of mathematical models. Agricultural systems, Amsterdam, v.89, n.2/3, p.225247, set. 2006. Disponível em: <http:/www.sciencedirect.com/ science/article/pii/S0308521X05002568>. Acesso em: 11 out. 2008. doi:10.1016/j.agsy.2005.11.004.
WAN, H.F. et al. Prediction of true metabolizable energy from chemical composition of wheat milling by-products for ducks. Poultry Science, Champaign, v.88, p.92-97, jan. 2009. Disponível em: $<$ http://ps.fass.org/content/88/1/92.full.pdf + html $>$. Acesso em: 20 dez. 2010. doi: 10.3382/ps.2008-00160.

ZHAO, F. et al. Predicting metabolizable energy of normal corn from its chemical composition in adult pekin ducks. Poultry Science, Champaign, v.87, p.1603-1608, ago. 2008. Disponível em: $<$ http://ps.fass.org/content/87/8/1603.full.pdf + html $>$. Acesso em: 20 dez. 2010. doi: 10.3382/ps.2007-00494.

ZONTA, M.C.M. et al. Energia metabolizável de ingredientes protéicos determinada pelo método de coleta total e por equações de predição. Ciência e Agrotecnologia, Lavras, v.28, n.6, p.14001407, nov./dez. 2004. Disponível em: <http://www.scielo.br/ scielo.php?pid=S1413-70542004000600024\&script $=$ sci_arttext $>$. Acesso em: 20 jun. 2009. doi: 10.1590/S1413-70542004000600023. 